\title{
Influência do ganho de peso sobre os níveis de glicemia casual: um estudo em crianças e adolescentes matriculados na rede pública de ensino na cidade de Salvador, BA, Brasil
}

\author{
Influence of weight gain on casual glucose levels: a study of children \\ and adolescents enrolled in public schools in Salvador, BA, Brazil
}

'Departamento de Ciência da Nutrição, Programa de PósGraduação em Alimentação e Nutrição, Escola de Nutrição, Universidade Federal da Bahia (UFBA), Salvador, BA, Brasil ${ }^{2}$ Programa de Pós-Graduação da Medicina e Saúde, Universidade Federal da Bahia (UFBA), Salvador, BA, Brasil

${ }^{3}$ Department of Epidemiology and Population Health, London School of Hygiene and Tropical Medicine (LSHTM), Keppel Street, London WC1E 7HT, UK

Correspondência para: Rita de Cássia Ribeiro Silva Rua Desembargador Oscar Dantas, 96, ap. 402 40150-260 - Salvador, BA, Brasil rcrsilva@ufba.br

Recebido em 28/9/2011 Aceito em $12 / 7 / 2012$
Rita de Cássia Ribeiro Silva', Ana Marlúcia Oliveira Assis', Luís Fernando Adan $^{2}$, Elizabete de Jesus Pinto ${ }^{2}$, Laura Cunha Rodrigues ${ }^{3}$

Gubstanciais mudanças vêm ocorrendo no padrão de saúde da população brasileira. A análise preliminar das tendências de indicadores dos níveis globais de saúde indica crescimento da ocorrência das doenças crônicas não degenerativas. $\mathrm{O}$ diabetes melito tipo 2 (DM), exemplo dessa situação, configura-se hoje como epidemia mundial, com um leque de fatores de risco conhecido, mas a definição e adoção de medidas de prevenção ainda representam grande desafio para os sistemas de saúde de todo o mundo. Dentre os fatores ambientais conhecidos que concorrem para o aumento do DM tipo 2, destacam-se as mudanças associadas ao estilo de vida, em que se incluem hábitos alimentares inadequados, inatividade física, uso abusivo do álcool e tabagismo. Os hábitos comportamentais correlacionam ainda com a obesidade, um dos principais preditores da DM. Na atualidade, a prevalência de excesso de peso entre crianças e adolescentes do Brasil varia de $20 \%$ a $35 \%$ (1), configurando-se importante fator de risco de DM nesse ciclo de vida $(2,3)$. A escassez de estudos epidemiológicos que avaliam a magnitude do diabetes e seus fatores associados nesse ciclo de vida impede a definição e a adoção de programas de saúde de caráter preventivo.

Assim, o efeito do ganho de peso sobre os níveis de glicemia casual foi avaliado em rastreamento para DM em estudantes da rede pública de ensino da cidade de Salvador, Bahia, Brasil. Os estudantes foram submetidos a dosagens de glicemias aferidas em amostras de sangue capilar, coletadas em tiras-teste $(\mathrm{mg} / \mathrm{dL})$, após digito-punctura com lancetadores automáticos (Roche Diagnostics $\mathrm{GmbH}$, Mannheim, Alemanha), com leitura efetuada pelo método de colorimetria por reflectância, em glicosímetros portáteis da marca Accutrend (Roche Diagnostics GmbH, Mannheim, Alemanha). Os casos suspeitos de diabetes foram detectados com glicemia capilar casual maior ou igual a $200 \mathrm{mg} / \mathrm{dL}$ (4). A partir das medidas de peso e altura, foi calculado o índice de massa corporal (IMC). Para avaliar o estado antropométrico, foram utilizadas como padrão de referência as tabelas de percentis da World Health Organization (WHO, 2007) segundo a idade (2 a 20 anos de idade) e o sexo (5). E, para classificação, utilizou-se a proposta da WHO, 2006 (6). A coleta de dados demográficos e econômicos da família foi feita utilizando-se de questionário estruturado. As informações demográficas dizem respeito ao sexo e à idade da criança. Para calcular o nível econômico dos escolares, utilizou-se o Critério de Classificação Econômica Brasil (CCEB), proposto pela Associação Brasi- 
leira das Empresas de Pesquisa (ABEP) (7). Construiu-se modelo de regressão linear múltipla, com erros normais, para estimar a associação entre o IMC e os níveis de glicemia casual, ajustada por variáveis demográficas e econômicas. Todos os testes estatísticos foram bicaudais. Para todos os testes, adotou-se o nível de significância de $5 \%$. O protocolo de estudo foi submetido ao Comitê de Ética do Instituto de Saúde Coletiva da Universidade Federal da Bahia, que avaliou e emitiu parecer favorável à sua realização. Dos 114.461 estudantes matriculados na rede pública municipal de ensino da cidade de Salvador, no ano de 2006 (dados fornecidos pela Secretaria de Educação e Cultura/SMEC do município), 38.637 estudantes, o equivalente a $34 \%$, foram submetidos ao exame de punção digital para screening de DM; os demais estudantes não apresentaram autorização dos pais para participarem do estudo. A prevalência de suspeitos de diabetes foi de $0,1 \%$. Verificou-se ainda que $0,8 \%$ dos estudantes apresentaram glicemia casual entre 140 a 199 $\mathrm{mg} / \mathrm{dL}$. A média da glicemia foi de $94,3 \pm 15,6 \mathrm{mg} /$ $\mathrm{dL}$, sugerindo uma discreta assimetria positiva. A média da glicemia casual foi significativamente mais elevada entre os meninos do que entre as meninas $(\mathrm{p}<0,001)$. Utilizando-se a técnica da regressão linear, identificou-se associação positiva e estatisticamente significante entre IMC e glicemia casual, mesmo após os ajustes por variáveis demográficas (sexo e idade) e situação econômica ( $\beta$ = 0,296; IC95\% 0,021 - 0,572) (Tabela 1). Embora a produção atual do conhecimento ainda indique que as informações sobre a ocorrência desse evento, nesta faixa etária, sejam escassas no Brasil, é possível supor, face ao aumento da prevalência de sobrepeso e obesidade, nesta etapa da vida, que essas estatísticas se aproximam daquelas evidenciadas em países desenvolvidos (8). Os resultados do presente estudo revelaram ainda tendência de aumento crescente dos níveis glicêmicos com o aumento do IMC. Essa tendência é também relatada em inúmeros estudos epidemiológicos, nacionais e internacionais, tanto em adultos quanto em crianças e adolescentes $(9,10)$. Nesses estudos, é reafirmado o papel deletério do excesso de peso na alteração dos níveis glicêmicos. Alguns autores sustentam a hipótese da resistência à insulina/ exaustão pancreática: a resistência crônica à insulina levaria a uma hipersecreção de insulina, com exaustão das células beta e consequente hiperglicemia (11). Assim, é possível supor que um modelo dietético nutricionalmente adequado, controle do peso corpóreo e redução do sedentarismo possam indiretamente contribuir com a adequação dos níveis glicêmicos dos indivíduos. Seguramente, a detecção precoce dessas alterações pode contri-
Tabela 1. Associação entre índice de massa corpórea e glicemia casual de crianças e adolescentes do ensino fundamental da rede pública do município de Salvador, Bahia, Brasil, 2006

\begin{tabular}{lccccc}
\hline \multirow{2}{*}{ Variáveis } & \multicolumn{2}{c}{$\begin{array}{c}\text { Modelo univariado } \\
\text { (bruto) }\end{array}$} & & \multicolumn{2}{c}{ Modelo completo (ajustado) } \\
\cline { 2 - 3 } \cline { 5 - 6 } & $\beta$ & \multicolumn{1}{|c}{$\mathbf{I C 9 5 \%}$} & & $\beta$ & IC95\% \\
\hline IMC & 0,283 & $0,009-0,557$ & & 0,296 & $0,021,0,572$ \\
Idade & & & & $-0,913$ & $-1,132,-0,694$ \\
Sexo & & & & $-2,727$ & $-3,048,-2,406$ \\
Situação & & & & 0,256 & $0,074,0,438$ \\
econômica & & & & \\
\hline
\end{tabular}

Categorias de referências: ${ }^{a}$ sexo feminino; ${ }^{b}$ classe $A+B$.

buir para o desenvolvimento de programas de saúde de caráter preventivo, especialmente no ambiente escolar, com enfoque na mudança do estilo de vida com vista à promoção da saúde e, assim, evitar o desenvolvimento prematuro do diabetes melito em jovens.

Suporte financeiro: Conselho Nacional de Desenvolvimento Científico e Tecnológico (CNPq) [processo n 402462/2005-0]; Coordenação de Aperfeiçoamento de Pessoal de Nível Superior (Capes) [processo: 1254/10-3].

Declaração: os autores declaram não haver conflitos de interesse científico neste estudo.

\section{REFERÊNCIAS}

1. Brazilian Institute of Geography and Statistics (2010). POF 20082009: Anthropometry and nutritional status of children, adolescents and adults in Brazil. Brazilian Institute of Geography and Statistics - IBGE [On-line]. [citado em 5 Mar, 2011]. Disponível em: URL: http://www.ibge.gov.br.

2. Gabbay M, Cesarini PR, Dib SA. [Type 2 diabetes in children and adolescents: literature review]. J Pediatr (Rio J). 2003;79(3):201-8.

3. Arslanian S. Type 2 diabetes in children: clinical aspects and risk factors. Horm Res. 2002;57 Suppl 1:19-28.

4. Type 2 diabetes in children and adolescents. American Diabetes Association. Diabetes Care. 2000;23(3):381-9.

5. de Onis M, Onyango AW, Borghi E, Siyam A, Nishida C, Siekmann J. Development of a WHO growth reference for school-aged children and adolescents. Bull World Health Organ. 2007;85(9):660-7.

6. WHO Multicentre Growth Reference Study Group. WHO Child Growth Standards: length/height-for-age w-f-a, weight-for-length, weight-for-height and body mass index-for-age: methods and development. Geneva: World Health Organization; 2006.

7. Associação Brasileira de Empresas de Pesquisa. Critério de Classificação Econômica Brasil. ABEP 2008 [citado em 2 Jul, 2009]. Disponível em: URL: http://www.abep.org.br.

8. Alberti G, Zimmet P, Shaw J, Bloomgarden Z, Kaufman F, Silink $M$. Type 2 diabetes in the young: the evolving epidemic: The International Diabetes Federation Consensus Workshop. Diabetes Care. 2004;27(7):1798-811.

9. Sartorelli DS, Franco LJ. [Trends in diabetes mellitus in Brazil: the role of the nutritional transition]. Cad Saude Publica. 2003;19 Suppl 1:S29-36.

10. Matyka KA. Type 2 diabetes in childhood: epidemiological and clinical aspects. Br Med Bull. 2008;86:59-75.

11. Cesaretti ML, KohImann Junior O. [Experimental models of insulin resistance and obesity: lessons learned]. Arq Bras Endocrinol Metabol. 2006;50(2):190-7. 